\title{
An intelligent parameter varying (IPV) approach for non-linear system identification of base excited structures
}

\author{
Soheil Saadat ${ }^{\mathrm{a}, *}$, Gregory D. Buckner ${ }^{\mathrm{a}}$, Tadatoshi Furukawa ${ }^{\mathrm{b}}$, Mohammad N. Noori ${ }^{\mathrm{a}}$ \\ ${ }^{a}$ Department of Mechanical \& Aerospace Engineering, North Carolina State University, North Carolina, NC 27695-7910, USA \\ ${ }^{\mathrm{b}}$ Department of Global Architecture, Graduate School of Engineering, Osaka University, 2-1 Yamadaoka, \\ Suita, Osaka 565-0871, Japan
}

\begin{abstract}
Health monitoring and damage detection strategies for base-excited structures typically rely on accurate models of the system dynamics. Restoring forces in these structures can exhibit highly non-linear characteristics, thus accurate non-linear system identification is critical. Parametric system identification approaches are commonly used, but require a priori knowledge of restoring force characteristics. Non-parametric approaches do not require this a priori information, but they typically lack direct associations between the model and the system dynamics, providing limited utility for health monitoring and damage detection. In this paper a novel system identification approach, the intelligent parameter varying (IPV) method, is used to identify constitutive non-linearities in structures subject to seismic excitations. IPV overcomes the limitations of traditional parametric and non-parametric approaches, while preserving the unique benefits of each. It uses embedded radial basis function networks to estimate the constitutive characteristics of inelastic and hysteretic restoring forces in a multi-degree-of-freedom structure. Simulation results are compared to those of a traditional parametric approach, the prediction error method. These results demonstrate the effectiveness of IPV in identifying highly non-linear restoring forces, without a priori information, while preserving a direct association with the structural dynamics.
\end{abstract}

Keywords: System identification; Artifical neural networks; Hysteresis; Nonlinear systems; Health monitoring

\section{Introduction}

Structures subjected to seismic excitations can exhibit non-linear behaviors such as inelastic and hysteretic restoring forces that cannot be accurately

\footnotetext{
${ }^{*}$ Corresponding author. Fax: +1-919-515-7968.

E-mail addresses: ssaadat@eos.ncsu.edu (S. Saadat), gbuckner@eos.ncsu.edu (G.D. Buckner), furukawa@ga.eng.osaka-u.ac.jp (T. Furukawa), mnoori@eos.ncsu.edu (M.N. Noori).
}

represented using linear, time-invariant models. For this reason, non-linear modeling and system identification are critical to the design, health monitoring, and damage detection of such structures. Both of these topics have been the focus of extensive research since the late 1960s. Noteworthy contributions have been made by Caughey [1], Iwan [2], Kobori, et al. [3], Beck [4], Baber and Wen [5], Toussi and Yao [6], Baber and Noori [7,8], Noori, et al. [9], Yar and Hammond [10,11], Noori, et al. [12], Peng and Iwan [13], Loh and Chung [14], Foliente, et al. [15], 


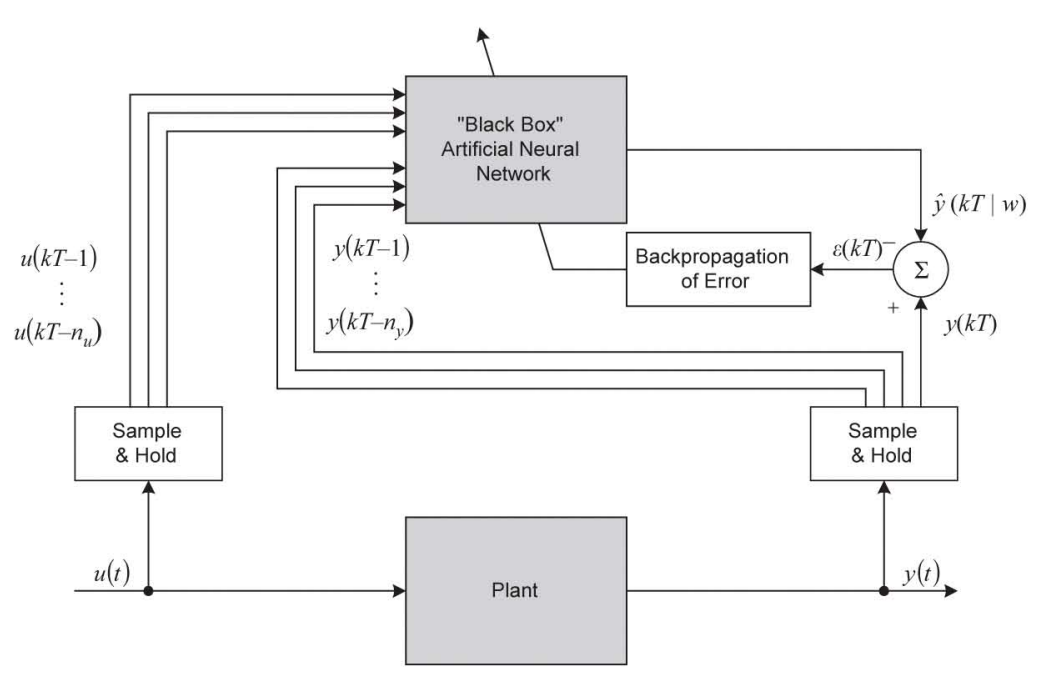

Fig. 1. "Black box" intelligent system identification.

Dobson, et al. [16], and others. Nevertheless, the characterization of inelastic and hysteretic restoring forces remains a challenging and relevant field of research.

Modeling and identification techniques can be categorized as being either parametric or non-parametric. Parametric system identification seeks to determine the "optimal" parameters for an assumed structural model such that modeled response closely matches the recorded response of the structure. Non-parametric techniques attempt to identify the "optimal" functional representation of the structure without any a priori assumptions about the model's structure.

Most of the published research involving the characterization of structural hysteresis has focused on parametric techniques. Parametric hysteresis models can be further categorized as those exhibiting either sharp yield transitions or smooth hysteresis loops. Sharp yield transition models are typically piecewise linear and include bi-linear models [1,17], tri-linear models [18], the Clough model [19], and the Q-hysteresis model [20]. These models are primarily used for dynamic response analysis of reinforced concrete and steel structures under seismic excitations [21]. The second category of parametric hysteresis models, that exhibit smooth hysteresis loops, include phenomenological models and its extensions that utilize additional state variables satisfying non-linear differential equations $[5,7,12,15,16,22]$. Parametric models have been used for a wide range of problems in structural dynamics.

Because of their unique capabilities in non-linear function approximation [23], artificial neural networks can be ideally suited for non-parametric modeling and system identification. The literature abounds with examples showing how "black box" artificial neural networks can be effectively used for non-parametric modeling, identification and control of non-linear dynamic systems [24-28]. Typically, "black box" neural networks are configured arbitrarily with a large number of system inputs and outputs, as shown in Fig. 1, and are trained to provide the complete non-linear mapping from the $m$-dimensional input space ( $m$ represents the number of excitation inputs) to the $r$-dimensional output space ( $r$ represents the number of output measurements). When artificial neural networks are implemented using this "black box" approach, little (if any) of the system information that might be obtained from traditional modeling techniques is utilized. Therefore, the associations between the neural network architecture and its weights to the underlying system dynamics and its parameters are rarely understood or utilized to improve the performance of the identification process.

A novel, hybrid approach to non-linear system identification combines the advantages of parametric models with the non-parametric capabilities of artificial 


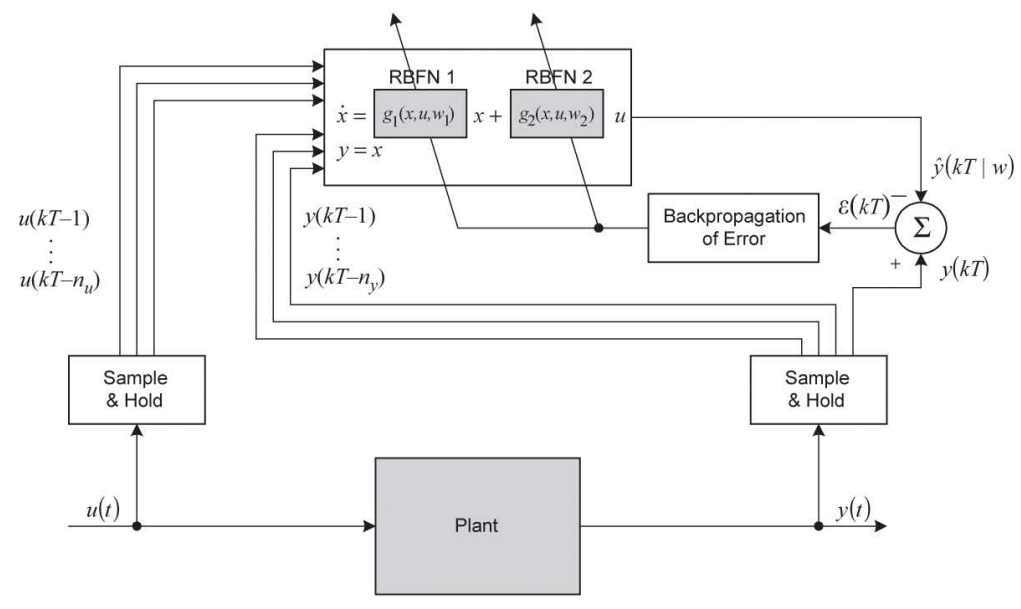

Fig. 2. IPV system identification.

neural networks. This "intelligent parameter varying" (IPV) approach to system identification incorporates artificial neural networks into a traditional parametric model. Artificial neural networks are used to identify the non-linear, time-varying portions of the system dynamics as shown in Fig. 2, in this case inelastic and hysteretic restoring forces that would be difficult or impossible to model using traditional approaches. The resulting model preserves a direct association between the neural network's architecture and its weights to the underlying system dynamics, and can be used both for design and health monitoring. This hybrid approach benefits from the wealth of research resulting from traditional modeling approaches, and simultaneously benefits from the non-linear adaptation and learning capabilities of artificial neural networks. Noteworthy contributions using this approach have been made by various researchers [29-32].

In this paper, the authors demonstrate the application of IPV modeling and identification techniques to the characterization of non-linear restoring forces in a multi-storey building subjected to seismic excitation. This approach is compared to a conventional parametric system identification approach for linear, bi-linear, and bi-linear hysteretic restoring forces.

\section{System modeling}

To study the dynamic response of a multi-storey building subjected to seismic inputs, a simple "shear building model" can be constructed by assuming that the building masses are lumped at each floor and that these floors are constrained to move laterally. Fig. 3 shows the three-storey shear building model used for this research. Note that each floor lumped mass $m_{i}$ represents the collective mass of the floor and its associated columns and beams, and that the springs and dampers represent the collective structural stiffness and damping between adjacent floors. Resulting lateral floor displacements represent the building's degrees of freedom and are represented by the state vector $\mathbf{x}=\left[x_{g}, x_{1}, x_{2}, x_{3}\right]^{\mathrm{T}}$.

In accordance with Newton's second law, the equations of lateral motion can be expressed:

$$
\begin{aligned}
& -f_{3}-c_{3}\left(\dot{x}_{3}-\dot{x}_{2}\right)=m_{3} \ddot{x}_{3}, \\
& -f_{2}-c_{2}\left(\dot{x}_{2}-\dot{x}_{1}\right)+f_{3}+c_{3}\left(\dot{x}_{3}-\dot{x}_{2}\right)=m_{2} \ddot{x}_{2}, \\
& -f_{1}-c_{1}\left(\dot{x}_{1}-\dot{x}_{g}\right)+f_{2}+c_{2}\left(\dot{x}_{2}-\dot{x}_{1}\right)=m_{1} \ddot{x}_{1},
\end{aligned}
$$

where $m_{1}, m_{2}, m_{3}$ represent the floors lumped masses, $c_{1}, c_{2}, c_{3}$ are constant structural damping coefficients, and $f_{1}, f_{2}, f_{3}$ are the inelastic stiffness restoring forces of the building. Alternately, these state equations can be expressed in terms of storey drifts $u_{1}, u_{2}, u_{3}$ :

$-f_{3}-c_{3}\left(\dot{u}_{3}-\dot{u}_{2}\right)-m_{3} \ddot{x}_{g}=m_{3} \ddot{u}_{3}$,

$$
\begin{aligned}
& -f_{2}-c_{2}\left(\dot{u}_{2}-\dot{u}_{1}\right)+f_{3}+c_{3}\left(\dot{u}_{3}-\dot{u}_{2}\right) \\
& -m_{2} \ddot{x}_{g}=m_{2} \ddot{u}_{2},
\end{aligned}
$$




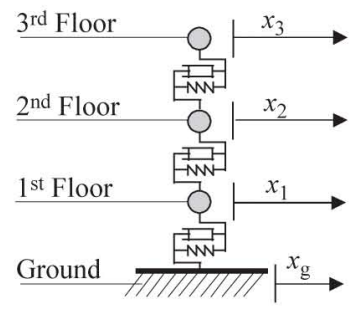

Fig. 3. Lumped-mass model of the three-storey shear building.

$$
\begin{aligned}
& -f_{1}-c_{1}\left(\dot{u}_{1}-\dot{u}_{g}\right)+f_{2}+c_{2}\left(\dot{u}_{2}-\dot{u}_{1}\right), \\
& -m_{1} \ddot{x}_{g}=m_{1} \ddot{u}_{1},
\end{aligned}
$$

where:

$u_{3}=x_{3}-x_{g}, \quad u_{2}=x_{2}-x_{g}, \quad u_{1}=x_{1}-x_{g}$.

Eq. (2) can be expressed in matrix form as

$$
\mathbf{M u ̈}+\mathbf{C u}=-\mathbf{M} \ddot{\mathbf{x}}_{g}-\mathbf{f}(\mathbf{x}, \mathbf{u}),
$$

where $\mathbf{M}$ and $\mathbf{C}$ are the diagonal mass and coupled damping matrices, respectively:

$$
\begin{aligned}
& \mathbf{M}=\left[\begin{array}{ccc}
m_{1} & 0 & 0 \\
0 & m_{2} & 0 \\
0 & 0 & m_{3}
\end{array}\right], \\
& \mathbf{C}=\left[\begin{array}{ccc}
c_{1}+c_{2} & -c_{2} & 0 \\
-c_{2} & c_{2}+c_{3} & -c_{3} \\
0 & -c_{3} & c_{3}
\end{array}\right] .
\end{aligned}
$$

\section{Parametric system identification}

For the purpose of parametric system identification, a dynamic system model is usually a linear, time-invariant difference equation structured in terms of an unknown parameter vector $\theta$. Once the structure of the model has been specified, various parameter estimation techniques can be employed to determine the optimal parameter vector. This process of parameter optimization uses recorded input-output system response data and attempts to minimize differences between the predicted output of the model $\hat{y}(t \mid \theta)$ and the actual response $y(t)$, the so-called prediction error [33]:

$\varepsilon(t, \theta)=y(t)-\hat{y}(t \mid \theta)$.

The optimal model among any set of candidate models parameterized in $\theta$ is thus the one that generates the smallest prediction error for a set of inputoutput data, as quantified by a suitable scalar-valued norm. The standard norm of choice is the quadratic norm, which is convenient both for computation and analysis and can be used to define the quadratic error cost function for $N$ data points:

$V_{N}(\theta)=\frac{1}{N} \sum_{t=1}^{N} \frac{1}{2} \varepsilon(t, \theta)^{2}$.

Techniques that seek to minimize prediction error norms such as (7) are collectively known as "prediction error methods", and include variants of the Least Squares Method, the Maximum Likelihood Method, and many others. An excellent introduction and coverage of these techniques is presented by Ljung [33]. Typically, these methods update the parameter vector analytically (a batch process) or numerically (an iterative process). Numerical approaches increment $\theta$ using information related to the error cost function at previous iterations:

$\theta(t+1)=\theta(t)+\alpha f(t)$.

Here $f$ is a search direction based on error cost function information acquired at previous iterations and $\alpha$ is a positive constant selected to provide the appropriate rate of decrease in the error cost function. These iterative techniques can be divided into three groups: (1) methods considering only the error cost function, (2) methods considering both the error cost function and its gradient, and (3) methods considering both the error cost function, its gradient, and its Hessian [33]. For the case of a system with a scalar output and a quadratic error cost function (the "non-linear least-squares problem"), a family of group 3 iterative search schemes is widely used:

$\theta(t+1)=\theta(t)-\mu\left[R_{N}\right]^{-1} V_{N}^{\prime}(\theta(t))$.

Here $\left[R_{N}\right]$ is a matrix that modifies the search direction. If $\left[R_{N}\right]$ is selected to be the identity matrix, the parameter estimation process (9) is known as a gradient descent method. If $\left[R_{N}\right]$ is selected to be the Hessian matrix, (9) becomes a Gauss-Newton 
method, and typically has better convergence close the optimum.

\section{Non-parametric system identification using radial basis function networks}

Non-parametric system identification using artificial neural networks ("black box" intelligent system identification) is essentially a multi-dimensional, non-linear regression problem. Consider the most general form of a non-linear plant with full state measurement:

$\dot{x}=f(x, u)$,

$y=x$.

Black box intelligent system identification provides a regression estimate $g(y, u, w)$ of the entire plant dynamics using past sampled outputs $y\left(t-1, \ldots, t-n_{a}\right)$ and inputs $u\left(t-1, \ldots, t-n_{b}\right)$. Analogous to prediction error methods, the network weights $w$ constitute a parameter vector that is iteratively modified in order to minimize differences between the predicted output $\hat{y}(t \mid w)$ and the actual response $y(t)$, the same prediction error defined in (6):

$\varepsilon(t, w)=y(t)-\hat{y}(t \mid w)$.

This network training process can be conducted off-line or on-line, and is illustrated in Fig. 1. The optimal set of network weights is thus the one that generates the smallest prediction error on unseen pairs of input-output measurements, as quantified by a suitable scalar-valued error cost function. Again the standard choice is to define a quadratic error cost function for $N$ data points:

$V_{N}(w)=\frac{1}{N} \sum_{t=1}^{N} \frac{1}{2} \varepsilon(t, w)^{2}$.

Techniques that seek to minimize prediction error norms such as (12) are collectively known as "backpropagation of error algorithms", and have been discussed extensively in the literature [23]. Analogous to the iterative parameter estimation techniques described in (8) and (9), the most general backpropagation techniques consider the error cost function, its gradient, and its Hessian to update the network weights [40]:

$w(t+1)=w(t)-\mu\left[R_{N}\right]^{-1} V_{N}^{\prime}(w(t))$.

As before, $\left[R_{N}\right]$ is a matrix that modifies the search direction. If $\left[R_{N}\right]$ is selected to be the identity matrix, the backpropagation process is known as a gradient descent method. If $\left[R_{N}\right]$ is selected to be the Hessian matrix, (13) becomes a Gauss-Newton method, and typically has better convergence close the optimum.

Literature abounds with variations of neural network architectures and activation functions for system identification; the most common architecture is a feedforward multi-layer network with hyperbolic tangent activation functions, the so-called multi-layer perceptron (MLP) [23]. However, the radial basis function network (RBFN) is frequently better suited to the task of system identification. The RBFN is a feedforward artificial neural network with a single, fully interconnected hidden layer [23]. The network uses multi-dimensional Gaussian (or radial basis) activation functions that, contrary to hyperbolic tangent functions, are localized with respect to the input space. As a result, parameter estimates obtained from a small region of the input space do not adversely affect estimates from other regions. The RBFN output is a weighted sum of hidden layer outputs, thus the learning algorithm is very simple and computationally inexpensive [23].

\section{Intelligent parameter varying system identification}

A novel, hybrid approach to non-linear system identification combines the advantages of parametric models with the non-parametric capabilities of artificial neural networks. This "intelligent parameter varying" (IPV) approach to system identification incorporates artificial neural networks into a traditional parametric model. Artificial neural networks are used to identify the non-linear, time-varying portions of the system dynamics that would be difficult or impossible to model using traditional approaches.

To illustrate this approach, consider a non-linear system represented by the linear parameter varying 
(LPV) model structure [34]:

$\dot{x}=f_{1}(x, u) \cdot x+f_{2}(x, u) \cdot u$,

$y=x$.

Here, the model structure is derived using traditional modeling approaches, but $f_{1}(x, u)$ and $f_{2}(x, u)$ represent unknown constitutive non-linearities. Parametric system identification approaches would require accurate, a priori representations of the non-linearities to obtain an optimal model for (14). In practice, these techniques might assume simplified linear representations, for example using constant parameters $a$ and $b$ :

$\dot{x}=a x+b u$

$y=x$

Because of their simple structures, parametric models such as (15) are appealing from computational point of view, but they lose accuracy as soon as the excursions in $x$ and $u$ go beyond the linear limits of the model.

In contrast, non-parametric system identification approaches, such as the "black box" implementation described in Section 4, do not require a priori representations of the non-linearities $f_{1}(x, u)$ and $f_{2}(x, u)$. Instead, these terms are usually lumped together into a single, more general, non-linear system form:

$\dot{x}=f(x, u)$,

$y=x$.

The "black box" identification of general, non-linear systems of the form (16) has been extensively studied and successfully implemented in areas such as control engineering. Since the "black box" implementation lumps together the system and the non-linearities $f_{1}(x, u)$ and $f_{2}(x, u)$ into a single model, therefore the "black box" model parameters are not traceable to the parameters of either the system or the non-linearities.

The IPV approach introduced here would preserve the model structure inherent in (14) without requiring a priori representations of the non-linearities $f_{1}(x, u)$ and $f_{2}(x, u)$. Instead, these terms would be represented by separate artificial neural networks $g_{1}\left(x, u, w_{1}\right)$ and $g_{2}\left(x, u, w_{2}\right)$ :

$\dot{x}=g_{1}\left(x, u, w_{1}\right) \cdot x+g_{2}\left(x, u, w_{2}\right) \cdot u$,

$y=x$.
By modeling the non-linearities $f_{1}(x, u)$ and $f_{2}(x, u)$ via separate artificial neural networks $g_{1}\left(x, u, w_{1}\right)$ and $g_{2}\left(x, u, w_{2}\right)$ the model structure of (17) is preserved. Therefore, the relation between model structure of (17) and artificial neural networks parameters is preserved.

This IPV model preserves the direct association between the neural network's architecture and its weights to the underlying system dynamics, and can be used both for design and health monitoring. This hybrid approach benefits from the wealth of research resulting from traditional modeling approaches, and simultaneously benefits from the non-linear adaptation and learning capabilities of artificial neural networks.

The networks $g_{1}\left(x, u, w_{1}\right)$ and $g_{2}\left(x, u, w_{2}\right)$ can be trained off-line or on-line using input-output data, and is illustrated in Fig. 2.

\section{Simulations}

In Section 6.1, a parametric system identification technique, the prediction error method, is applied to three-storey shear building model subject to earthquake excitations. Results are presented for three distinct cases: buildings with linear, bi-linear, and bi-linear hysteretic restoring forces. Then, in Section 6.2, the novel IPV approach is applied to these same cases. Advantages and practical limitations associated with both methods are discussed in Section 6.3.

\subsection{Parametric system identification of a shear building model}

As outlined in Section 2, the lateral dynamics of a three-storey structure subjected to earthquake excitations can be represented using the shear building model (1). To evaluate the effectiveness of parametric techniques in identifying the restoring forces of this building, a series of computer simulations was conducted. Three distinct cases of restoring forces (linear, bi-linear, and bi-linear hysteretic) were evaluated subject to seismic excitation. To facilitate these simulations, the shear-building model was parameterized using stiffness and yield displacement matrices $\mathbf{K}$ 


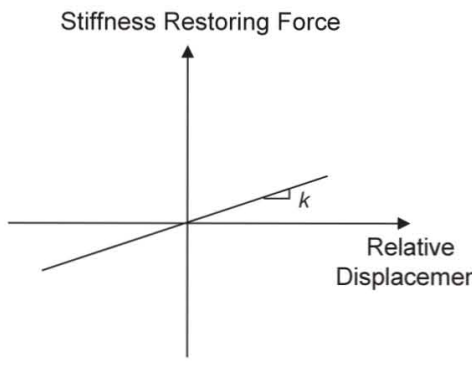

(a)

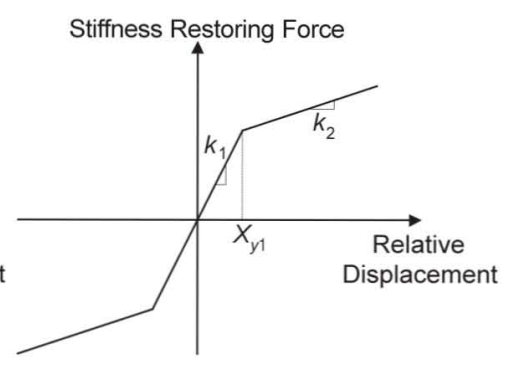

(b)

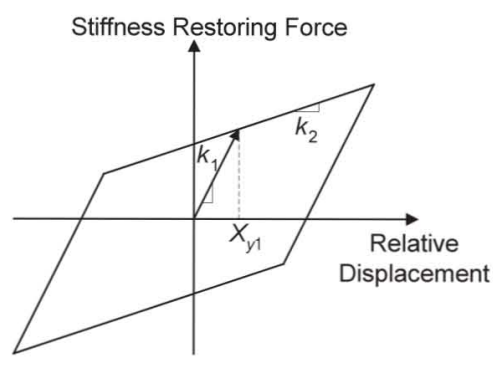

(c)

Fig. 4. Parametric models used for modeling the restoring force of the three-storey shear building, (a) linear, (b) bi-linear and (c) bi-linear hysteretic.

and $\mathbf{X}_{y}$ :

$\mathbf{K}=\left[\begin{array}{ll}k_{11} & k_{12} \\ k_{21} & k_{22} \\ k_{31} & k_{32}\end{array}\right], \quad \mathbf{X}_{y}=\left[\begin{array}{ll}x_{y_{11}} & x_{y_{12}} \\ x_{y_{21}} & x_{y_{22}} \\ x_{y_{31}} & x_{y_{32}}\end{array}\right]$.

The columns of the stiffness matrix represent the primary and secondary stiffnesses, while the rows correspond to building floors respectively. The columns and rows of the yield displacement matrix correspond similarly to the primary and secondary yield displacements and building floors. At the yield displacements, the stiffness and consequently the slope of the inelastic restoring force changes. Note that the yield displacements in the last column are assumed to be infinite. Thus, for linear restoring forces the yield displacement matrix has only one column with infinite entries. Fig. 4 shows the restoring force models used for simulation and parametric identification.

For each of the following cases, building response data was generated using the three-storey shear building model and fourth-order Runge-Kutta integration with a time-step of $0.01 \mathrm{~s}$. A standard parametric system identification approach, namely the Gauss-Newton method defined in Section 3, was implemented using Matlab's System ID toolbox with custom modifications for bi-linear and bi-linear hysteretic systems. This algorithm automatically terminated whenever the change in the error cost function (7) fell below $1 \%$ over two consecutive epochs. One epoch is defined to be a series of model parameter vector updates utilizing set of input-output "training" data.
The first case utilized linear restoring force models (Fig. 4a) to generate building response data. Constant stiffnesses of $8.0 \mathrm{~N} / \mathrm{m}$ were selected for all three floors. Floor masses were specified to be $5.0 \mathrm{~kg}$, and linear damping coefficients were set to $0.001 \mathrm{~N} \mathrm{~s} / \mathrm{m}$. The parametric system identification algorithm assumed these restoring forces to be linear, and the floor masses to be known. The initial estimates of $\hat{K}$ 's were distributed above and below the nominal values, as shown in Table 1. System identification took nine epochs to converge with a final error cost function of $1.8191 \mathrm{e}^{-87}$. Results of this simulation are presented in Table 1.

The second case utilized bi-linear restoring force models (Fig. 4b), with constant primary stiffnesses of $8.0 \mathrm{~N} / \mathrm{m}$, constant secondary stiffness of $2.0 \mathrm{~N} / \mathrm{m}$, and primary yield displacements of $0.02 \mathrm{~m}$ for all three floors. Floor masses and linear damping coefficients were again set to $5.0 \mathrm{~kg}$ and $0.001 \mathrm{~N} \mathrm{~s} / \mathrm{m}$, respectively. The parametric system identification algorithm assumed these restoring forces to be bi-linear, the floor masses and primary yield displacements to be known. The initial estimates of $\hat{K}$ 's were distributed above and below the nominal values, as shown in Table 2. System identification took nine epochs to converge with a final error cost function of $3.74274^{-87}$. Results of this simulation are presented in Table 2 .

The third and final case utilized bi-linear hysteretic restoring force models ( Fig. 4c), with constant primary stiffnesses of $8.0 \mathrm{~N} / \mathrm{m}$, constant secondary stiffness of $2.0 \mathrm{~N} / \mathrm{m}$, and primary yield displacements of $0.02 \mathrm{~m}$ for all three floors. Floor masses and linear damping coefficients were again set to $5.0 \mathrm{~kg}$ and $0.001 \mathrm{Ns} / \mathrm{m}$, 
Results of parametric system identification (Gauss-Newton) for building with linear restoring forces

\begin{tabular}{lllllll}
\hline & \multicolumn{2}{l}{ Exact } & & & \multicolumn{2}{c}{ Estimated } \\
\cline { 2 - 3 } \cline { 6 - 6 } & $M$ & $C$ & $K_{1}$ & & Initial $\hat{K}_{1}$ & 8.0 \\
First floor & 5 & 0.001 & 8.0 & 6.5 & 8.0 \\
Second floor & 5 & 0.001 & 8.0 & 7 & 8.0 \\
Third floor & 5 & 0.001 & 8.0 & 9.5 & 8.0 \\
\hline
\end{tabular}

Table 2

Results of parametric system identification (Gauss-Newton) for building with bi-linear restoring forces-primary yield displacement of $0.02 \mathrm{~m}$

\begin{tabular}{|c|c|c|c|c|c|c|c|c|}
\hline & \multicolumn{4}{|c|}{ Exact } & \multicolumn{4}{|l|}{ Estimated } \\
\hline & $M$ & $C$ & $K_{1}$ & $K_{2}$ & Initial $\hat{K}_{1}$ & Final $\hat{K}_{1}$ & Initial $\hat{K}_{2}$ & Final $\hat{K}_{2}$ \\
\hline First floor & 5 & 0.001 & 8.0 & 2.0 & 8.5 & 8.0 & 2.3 & 2.0 \\
\hline Second floor & 5 & 0.001 & 8.0 & 2.0 & 7.7 & 8.0 & 1.7 & 2.0 \\
\hline Third floor & 5 & 0.001 & 8.0 & 2.0 & 8.4 & 8.0 & 2.2 & 2.0 \\
\hline
\end{tabular}

Table 3

Results of parametric system identification (Gauss-Newton) for building with bi-linear hysteretic restoring forces-primary yield displacement of $0.02 \mathrm{~m}$

\begin{tabular}{|c|c|c|c|c|c|c|c|c|}
\hline & \multicolumn{4}{|c|}{ Exact } & \multicolumn{4}{|l|}{ Estimated } \\
\hline & $M$ & $C$ & $K_{1}$ & $K_{2}$ & Initial $\hat{K}_{1}$ & Final $\hat{K}_{1}$ & Initial $\hat{K}_{2}$ & Final $\hat{K}_{2}$ \\
\hline First floor & 5 & 0.001 & 8.0 & 2.0 & 8.5 & 8.0 & 2.3 & 2.0 \\
\hline Second floor & 5 & 0.001 & 8.0 & 2.0 & 7.7 & 8.0 & 1.7 & 2.0 \\
\hline Third floor & 5 & 0.001 & 8.0 & 2.0 & 8.4 & 8.0 & 2.2 & 2.0 \\
\hline
\end{tabular}

respectively. The parametric system identification algorithm assumed these restoring forces to be bi-linear hysteretic, the floor masses and primary yield displacements to be known. The initial estimates of $\hat{K}$ 's were distributed above and below the nominal values, as shown in Table 3. System identification took six epochs to converge with a final error cost function of $4.33094^{-90}$. Results of this simulation are presented in Table 3.

\subsection{IPV system identification of a shear building model}

To evaluate the effectiveness of the IPV approach in identifying restoring forces, a second series of com- puter simulations was conducted. The same three cases of restoring forces (linear, bi-linear, and bi-linear hysteretic) were evaluated subject to seismic excitation.

For the three-storey shear building model (1), the stiffness and damping terms can be lumped together as net restoring forces $R_{1}, R_{2}, R_{3}$ :

$$
\begin{aligned}
& -R_{3}-m_{3} \ddot{x}_{g}=m_{3} \ddot{u}_{3}, \\
& -R_{2}+R_{3}-m_{2} \ddot{x}_{g}=m_{2} \ddot{u}_{2}, \\
& -R_{1}+R_{2}-m_{1} \ddot{x}_{g}=m_{1} \ddot{u}_{1} .
\end{aligned}
$$

Using the IPV approach outlined in Section 5, three separate RBFNs $g_{1}, g_{2}, g_{3}$ were used to model these net restoring forces. These RBFN inputs result directly 


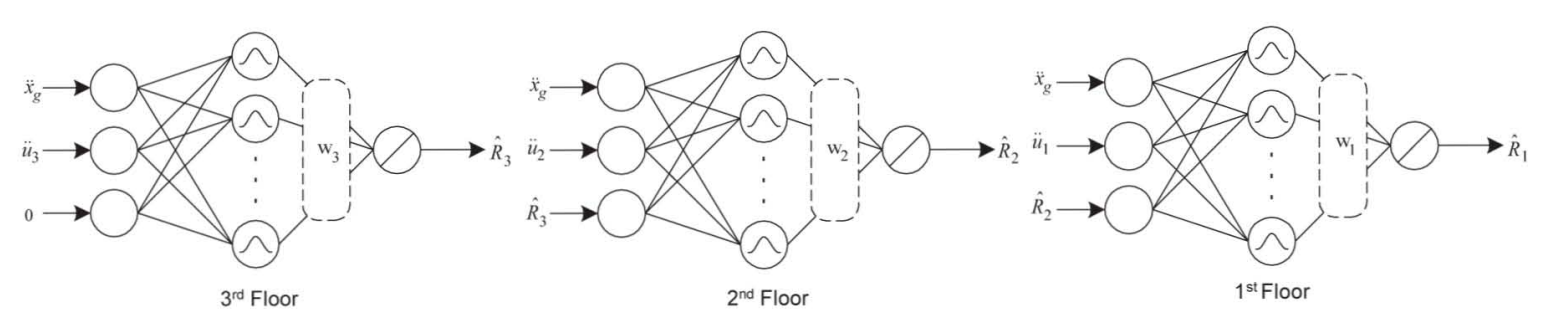

Fig. 5. Radial basis function networks used for modeling the restoring forces of the building.

from the equations of lateral motion (19):

$\hat{R}_{3}=g_{3}\left(\ddot{u}_{3}, \ddot{x}_{g}\right)$,

$\hat{R}_{2}=g_{2}\left(\ddot{u}_{2}, \ddot{x}_{g}, \hat{R}_{3}\right)$,

$\hat{R}_{1}=g_{1}\left(\ddot{u}_{1}, \ddot{x}_{g}, \hat{R}_{2}\right)$.

Fig. 5 shows the structure of the RBFNs used. The response data from the previous cases was used to "train" each of these neural networks in a systematic manner. First, acceleration data from the building's base and third floor were used to identify the net restoring force of the third floor $\hat{R}_{3}=g_{3}\left(\ddot{u}_{3}, \ddot{x}_{g}\right)$. Next, this restoring force estimate, combined with acceleration data from the building's base, second and third floors, was used to identify the net restoring force of the second floor $\hat{R}_{2}=g_{2}\left(\ddot{u}_{2}, \ddot{x}_{g}, \hat{R}_{3}\right)$. Finally, this restoring force estimate, combined with acceleration data from the building's base, first and second floors, was used to identify the net restoring force of the first floor $R_{1}=g_{1}\left(\ddot{u}_{1}, \ddot{x}_{g}, \hat{R}_{2}\right)$.

The inputs to each RBFN were normalized, and three activation functions were uniformly distributed along each dimension of the input space $(-0.25,0.50$, and 1.25), resulting in 27 activation functions for each RBFN. The spread of each activation function were set to 5 , and the weights were initially set to zero. These weights were updated incrementally using a "training set" consisting of randomly-selected input-output response data $(50 \%$ of the entire simulation data). Training continued until the change in the error cost function (12) for a "testing set" (the remaining 50\% of simulation data) fell below $1 \%$ over two consecutive epochs. Learning rates of 20, 40 and 60 are used for the first, second and third floor restoring force networks, respectively.

The first IPV case utilized the same linear restoring force models and building parameters used for parametric system identification (see Table 1). System identification took 5,10 , and 5 epochs for the third, second, and first floors to converge with a final error cost functions of $6.57191 \mathrm{e}^{-6}, 8.88639 \mathrm{e}^{-4}$, and $2.66114 \mathrm{e}^{-3}$, respectively. Case results are presented in Fig. 6. The identified restoring forces in this figure are so close to the actual restoring forces that it is difficult to distinguish the two. It is important to note that, in contrast to the parametric results of Section 6.1, IPV identified these restoring forces without a priori characterization: these forces were not assumed to be linear, and the initial RBFN weights were set to zero (not distributed about the "actual" values).

The second IPV case utilized the same bi-linear restoring force models and building parameters used for parametric system identification (see Table 2). System identification took 35,8 , and 13 epochs for the third, second, and first floors to converge to final error cost functions of $4.31392 \mathrm{e}^{-7}, 2.37501 \mathrm{e}^{-4}$, and $5.43597 \mathrm{e}^{-4}$, respectively. Case results are presented in Fig. 7, where again it is difficult to distinguish the identified restoring forces from the actual restoring forces. As before, these estimated restoring forces were identified without a priori characterization of any kind.

The third IPV case utilized the same hysteretic restoring force models and building parameters used for parametric system identification (see Table 3). System identification took 46, 11, and 15 epochs for the third, second, and first floors to converge to final error cost functions of $1.20927 \mathrm{e}^{-7}, 1.17006 \mathrm{e}^{-4}$, and $1.33650 \mathrm{e}^{-4}$, respectively. Case results are presented in Fig. 8. Even for this most challenging case, the estimated restoring forces (identified without a priori characterization) are nearly indistinguishable from the actual values. 

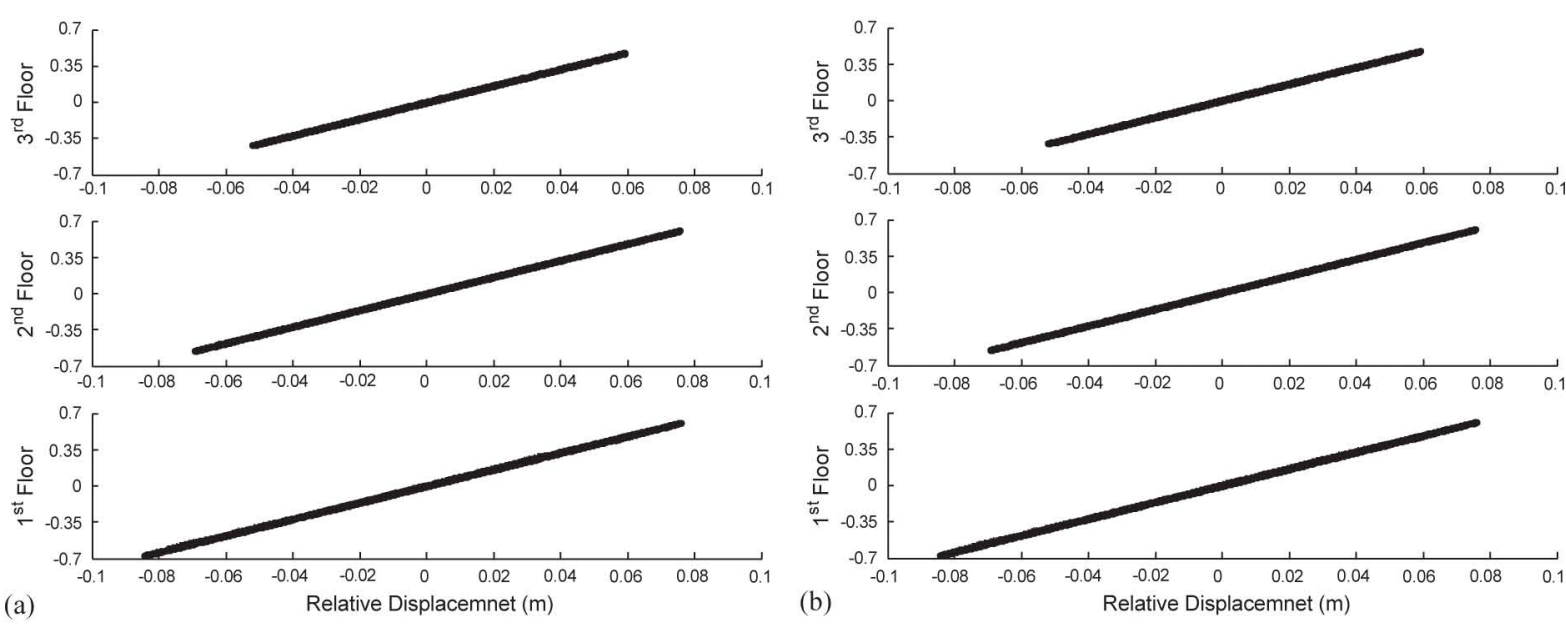

Fig. 6. Estimated $(+)$ versus Exact $(\circ)$ net restoring forces $(N)$ for a three-storey shear building with linear restoring force model: (a) training set and (b) testing set.
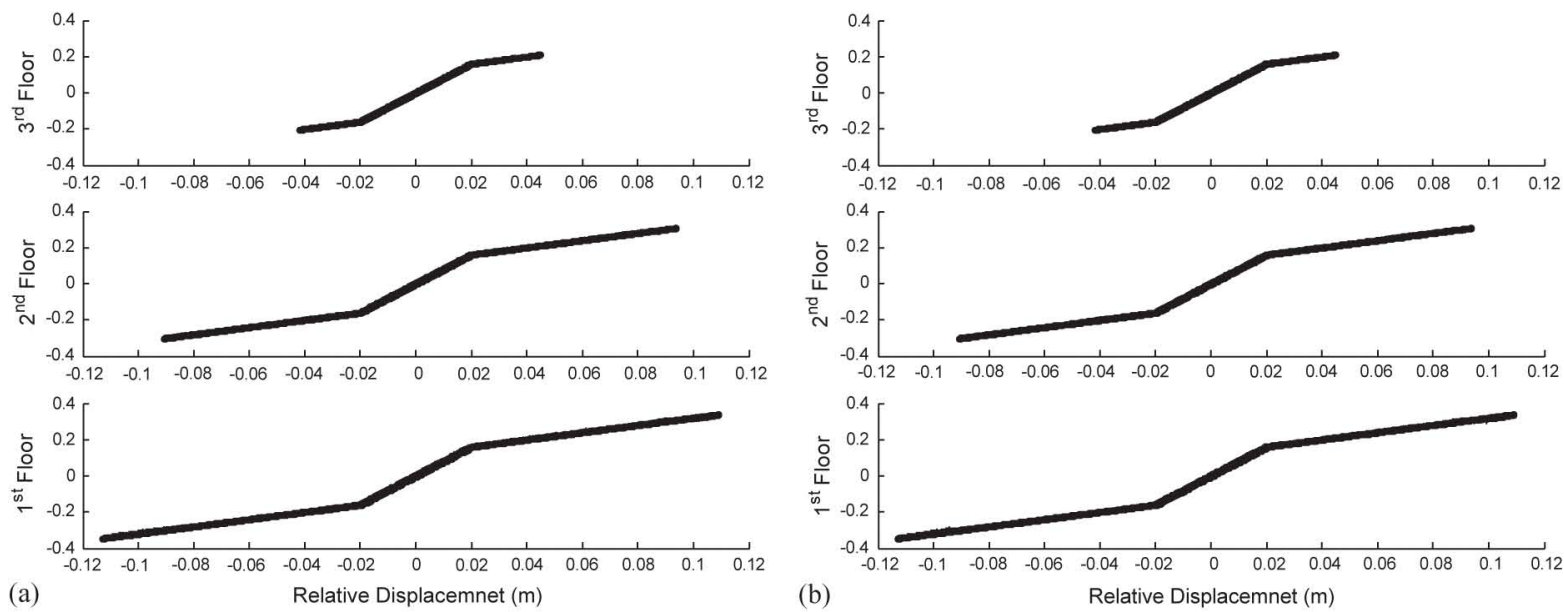

Fig. 7. Estimated $(+)$ versus Exact $(0)$ net restoring forces $(N)$ of building with bi-linear restoring force model: (a) training set and (b) testing set.

\subsection{Results: IPV system identification versus parametric system identification}

The simulation results of Section 6.1 and 6.2 clearly illustrate the benefits of IPV system identification for non-linear systems versus conventional parametric approaches. First, the IPV approach does not require a priori knowledge of the constitutive non-linearities in (14), as the Prediction Error Methods do. Hence it can be used to model and identify completely general

non-linear systems. Furthermore, IPV does not suffer from model "over parameterization", and can be applied to systems with multiple degrees of freedom. For example, in Section 6.1 the yield displacements associated with each primary stiffness were assumed to be known, otherwise the parametric system identification would produce erroneous results, while in Section 6.2 no assumptions were made regarding the structure of these non-linearities. Finally, each simulation of Section 6.1 required approximately ten hours of CPU time 

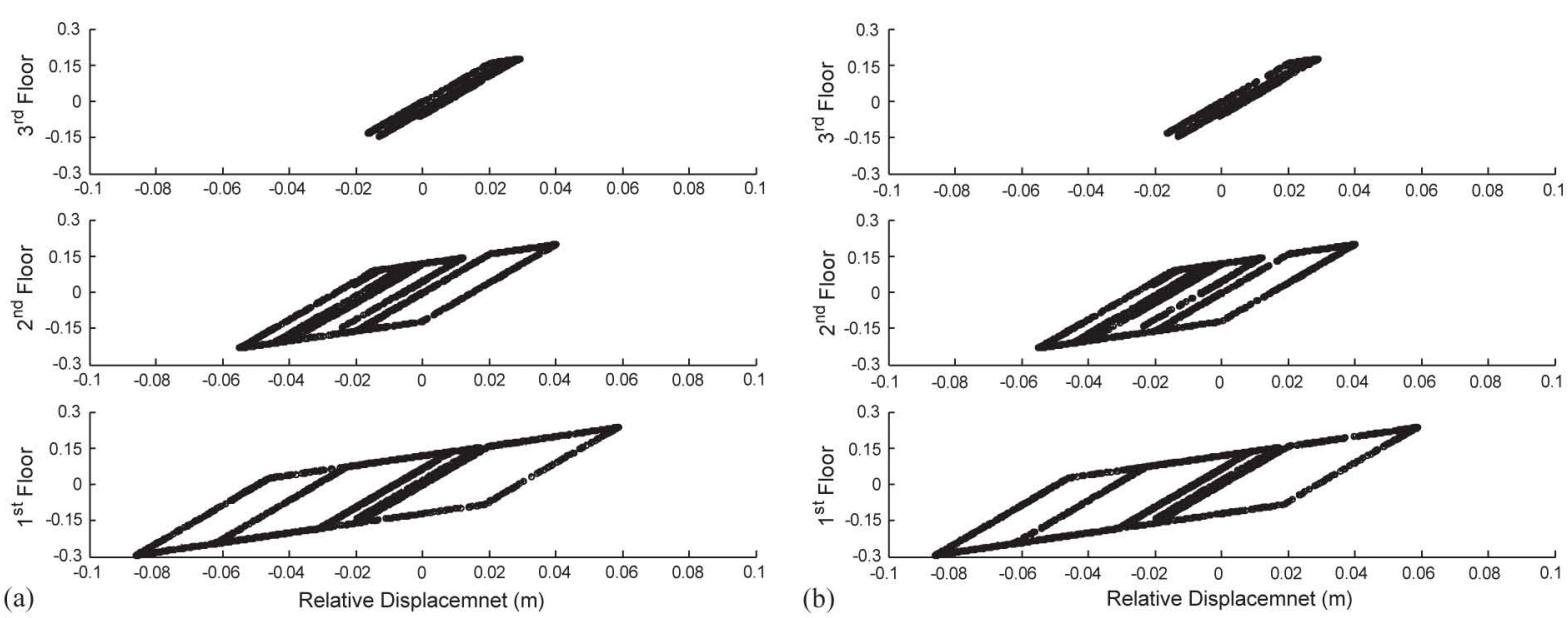

Fig. 8. Estimated $(+)$ versus Exact $(0)$ net restoring forces $(N)$ of building with hysteretic restoring force model: (a) training set and (b) testing set.

to complete, primarily due to the large number of function evaluations needed to calculate search directions in Eq. (9). Each simulation of Section 6.2 was completed in less than nine minutes of CPU time, therefore IPV is significantly cheaper computationally.

\section{Conclusion}

Parametric system identification approaches provide direct associations between the model and the system dynamics, but require a priori knowledge of constitutive non-linearities. Non-parametric approaches lack direct associations between the model and the system dynamics, but do not require a priori knowledge of constitutive non-linearities. A simple yet general approach that overcomes the intrinsic limitations of traditional parametric and non-parametric approaches, while preserving their unique benefits of each, is presented. Simulations reveal that this IPV approach effectively identifies highly non-linear restoring forces without a priori knowledge of their constitutive characteristics. The simplicity of this approach, combined with its adaptive capabilities to approximate and generalize non-linear information, make it ideally suited to on-line health monitoring and damage detection applications. Although the shear building model used in this study represents a simple chain-like structure, the concept of the IPV approach should be applicable to other more general dynamic systems with custom modifications. In any dynamic system, the IPV approach tries to identify the non-linear time-varying portions of the system dynamics, based on a loosely defined system model, in terms of response data or other measurable quantities. Effects of measurement noise and incomplete data on the performance of IPV-based system identification are issues that will be addressed in subsequent studies.

\section{Acknowledgements}

The research reported in this paper was conducted within the framework of the US-Japan Cooperative Research on Smart Structures and Structural Health Monitoring with Disaster Prevention Research Institute (DPRI) of Japan. This research was supported by grants from the National Sea Grants Industrial Fellowship Program, and R.J. Reynolds endowed funds.

\section{References}

[1] T.K. Caughey, Random excitation of a system with bilinear hysteresis, J. Appl. Mech. ASME 27 (1960) 649-652.

[2] W.D. Iwan, A distributed-element model for hysteresis and its steady-state dynamic response, J. Appl. Mech. ASME 33 (4) (1966) 612-617. 
[3] T. Kobori, R. Minai, Y. Suzuki, Stochastic Seismic Response of Hysteresis Structures Part I, Bull Disaster Prevention Research Institute 26 (1976) 55-70.

[4] J.L. Beck, Determining models of structures from earthquake records, Ph.D. Dissertation, California Institute of Technology, Pasadena, CA, 1978.

[5] T.T. Baber, Y.K. Wen, Random vibration of hysteretic degrading systems, ASCE J. Eng. Mech. Division 107 (6) (1981) 1069-1087.

[6] S. Toussi, J.T.P. Yao, Hysteretic identification of existing structures, ASCE J. Eng. Mech. Division 109 (5) (1983) 1189-1202.

[7] T.T. Baber, M.N. Noori, Random vibration of pinching, hysteretic systems, ASCE J. Eng. Mech. Division 110 (7) (1984) 1036-1049.

[8] T.T. Baber, M.N. Noori, Modeling general hysteresis behavior and random vibration application, ASME Design Engineering Division Conference and Exhibit on Mechanical Vibration and Noise, Cincinnati, Ohio, 85-DET-181, 1985.

[9] M.N. Noori, H. Davoodi, J.D. Choi, Zero and nonzero mean random vibration analysis of a new general hysteresis model, J. Probab. Eng. Mech. 1 (4) (1987) 192-201.

[10] M. Yar, J.K. Hammond, Modeling and response of bilinear hysteretic systems, ASCE J. Eng. Mech. 113 (1987) 1000-1013.

[11] M. Yar, J.K. Hammond, Parameter estimation for hysteretic systems, J. Sound Vib. 117 (1) (1987) 161-172.

[12] M.N. Noori, M. Saffar, R. Christodoulidu, Equivalent linearization of a newly introduced general hysteretic model, in: P.D. Spanos, C.A. Brebbia (Eds.), Proceedings of the First international Conference on Computational Stochastic Mechanics, Computational Mechanics, Kerkyra, Greece, 1991, pp. 313-326.

[13] C.Y. Peng, W.D. Iwan, An identification methodology for a class of hysteretic structures, Earthquake Eng. Struct. Dyn. 21 (1992) 695-712.

[14] C. Loh, S. Chung, A three-stage identification approach for hysteretic systems, Earthquake Eng. Struct. Dyn. 22 (1993) 129-150.

[15] G. Floiente, M.P. Singh, M.N. Noori, Equivalent linearization of generally pinching hysteretic degrading systems, Earthquake Eng. Struct. Dyn. 25 (1996) 611-629.

[16] S. Dobson, M.N. Noori, Z. Hou, Y. Suzuki, Boolean modeling of mechanical hysteretic systems, Struct. Safety Reliability (1996) 779-785.

[17] T.K. Caughey, Sinusoidal excitation of a system with bilinear hysteresis, J. Appl. Mech. Trans. ASME 27 (1960) 640-643.

[18] T. Takeda, M.A. Sozen, N.N. Nielsoen, Reinforced concrete response to simulated earthquakes, ASCE J. Struct. Division 96 (ST12) (1970) 2557-2573.

[19] Y.S. Clough, S.B. Johnson, Effect of stiffness degradation on earthquake ductility requirements, Transactions of Japan
Earthquake Engineering Symposium, Tokyo, Japan, 1996, pp. 227-232.

[20] M. Saiidi, M.A. Sozen, Simple and complex models for nonlinear seismic response of reinforced concrete structures, SRS-465, Civil Engineering Studies, University of Illinois, Urbana, U.S.A., 1979.

[21] C.-H. Loh, R.-C. Ho, Seismic damage assessment based on different hysteretic rules, Earthquake Engineering and Structural Dynamics 19 (1990) 753-771.

[22] Y.K. Wen, Method for random vibration of hysteretic systems, ASCE J. Eng. Mech. Division 102 (2) (1976) 249-263.

[23] S.S. Haykin, Neural networks: a comprehensive foundation, Prentice-Hall, Upper Saddle River, NJ, 1999.

[24] S.F. Masri, T.K. Caughey, A nonparametric identification technique for nonlinear dynamic problems, J. Appl. Mech. 46 (1979) 433-445.

[25] K.S. Narendra, K. Parthasarathy, Identification and control of dynamical systems using neural networks, IEEE Trans. Neural Networks 1 (1) (1990) 4-27.

[26] S. Chen, S.A. Billings, Neural networks for nonlinear dynamic system modeling and identification, Int. J. Control 56 (2) (1992) 319-346.

[27] S. Lu, T. Basar, Robust nonlinear system identification using neural network models, IEEE Trans. Neural Networks 9 (3) (1998) 1-24.

[28] A.G. Chassiakos, S.F. Masri, Neural net based identification of structural systems, IMACS Parallel and Distributed Computing in Engineering Systems, Elsevier, Amsterdam, 1992, pp. 371-375.

[29] S.F. Masri, A.G. Chassiakos, T.K. Caughey, Structureunknown non-linear dynamic systems: identification through neural networks, Smart Mater. Struct. 1 (1992) 45-56.

[30] J. Suontausta, V.T. Ruoppila, H.N. Koive, Modeling of non-linear systems using radial basis function networks, IFAC System Identification, Vol. 2, Copenhagen, Denmark, 1994, pp. 627-632.

[31] G.D. Buckner, K.T. Schuetze, J.H. Beno, Active vehicle suspension control using intelligent feedback linearization, Proceedings of the American Controls Conference, Vol. 6, Chicago, IL, 2000, pp. 4014-4018.

[32] G.D. Buckner, K.T. Schuetze, J.H. Beno, Intelligent feedback linearization for active vehicle suspension control, ASME J. Dynamic Syst. Measure. Control 123 (4) (2001) 727-733.

[33] J. Ljung, System Identification, Theory for the User, 2nd Edition, Prentice-Hall, Inc, Englewood Cliffs, NJ, 1999.

[34] J.S. Shamma, Linear parameter varying systems, The Control Handbook, CRC Press, Boca Raton, 1996, pp. 395-396. 